\title{
Looking for an International Consensus Exercise Guidelines in HIV
}

\section{Nuria Garatachea*}

Department of Physiotherapy and Nursing, University of Zaragoza, Ronda Misericordia, Spain

Nowadays Human Immunodeficiency Virus (HIV) is considered as a chronic disease because individuals have the potential to live upward of 20 years on highly active antiretroviral therapy. Disease process could culminate in HIV-related disability that has been associated with fatigue and decreased physical function in gas well as other factors that may limit ability of people to carry out necessary daily life activities.

Decreased metabolic, muscular, and aerobic capacity in people with HIV appears to have an important role in the mechanism of fatigue and physical disability. Physiological deconditioning also may play a role in life activities. Thus, physical activity or exercise program could be a beneficial treatment for prevent or improve HIV-related disability.

Evidence for an achievable aerobic and strength positive training effect have been documented in the literature. Although, it has been demonstrated that physical activity has many physical and mental benefits in people who are living with HIV, there are no unique exercise guidelines for that population.
We keep in mind that this lack of unique exercise guidelines could be for the limitation that includes the absence of direct evidence for the link between physiological alterations and exercise and physical activity limitations in people with HIV. The complex nature of HIV disease also may influence interpretation of the pathophysiological and exercise limitation data in this population.

In short, until now exercise guidelines do not exist for people who are living with VIH, with specific and clear recommendations in terms of: duration, intensity, frequency, and mode. Although some efforts have been done, it is insufficient. I would like to make a call to the main recognized associations of sport medicine, exercise science and/or VIH appealing to their sense of responsibility in this topic. I hope and believe that intense and exceptional efforts by teams of researchers, clinicians, exercise professionals among others have profitable benefits, in order to achieve an international consensus exercise guidelines to improve physical and mental functioning in VIH-infected in next future.
${ }^{*}$ Corresponding author: Nuria Garatachea, PhD, Faculty of Health and Sport Science, Department of Physiotherapy and Nursing, University of Zaragoza, Ronda Misericordia 5. 22001-Huesca, Spain, Tel: +34-976761000; Fax: +34-976761720; E-mail: nuria.garatachea@unizar.es

Received January 24, 2012; Accepted January 26, 2012; Published January 30 2012

Citation: Garatachea N (2012) Looking for an International Consensus Exercise Guidelines in HIV. J AIDS Clinic Res 3:e103. doi:10.4172/2155-6113.1000e103

Copyright: ( 2012 Garatachea N. This is an open-access article distributed under the terms of the Creative Commons Attribution License, which permits unrestricted use, distribution, and reproduction in any medium, provided the original author and source are credited. 